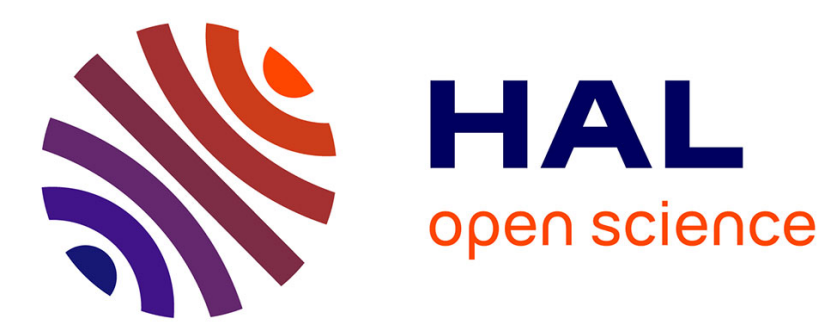

\title{
Numerical porosimetry: Evaluation and comparison of yield stress fluids method, mercury intrusion porosimetry and pore network modelling approaches
}

Antonio Rodriguez de Castro, Mehrez Agnaou, Azita Ahmadi-Senichault, Abdelaziz Omari

\section{To cite this version:}

Antonio Rodriguez de Castro, Mehrez Agnaou, Azita Ahmadi-Senichault, Abdelaziz Omari. Numerical porosimetry: Evaluation and comparison of yield stress fluids method, mercury intrusion porosimetry and pore network modelling approaches. Computers \& Chemical Engineering, 2020, 133, pp.106662. 10.1016/j.compchemeng.2019.106662 . hal-02509698

\section{HAL Id: hal-02509698 \\ https://hal.science/hal-02509698}

Submitted on 23 Jul 2021

HAL is a multi-disciplinary open access archive for the deposit and dissemination of scientific research documents, whether they are published or not. The documents may come from teaching and research institutions in France or abroad, or from public or private research centers.
L'archive ouverte pluridisciplinaire HAL, est destinée au dépôt et à la diffusion de documents scientifiques de niveau recherche, publiés ou non, émanant des établissements d'enseignement et de recherche français ou étrangers, des laboratoires publics ou privés. 


\title{
Numerical porosimetry: Evaluation and comparison of yield stress fluids method, mercury intrusion porosimetry and pore network modelling approaches
}

\author{
Antonio Rodríguez de Castro ${ }^{\mathrm{a}, *}$, Mehrez Agnaou ${ }^{\mathrm{b}}$, Azita Ahmadi-Sénichault ${ }^{\mathrm{c}}$, \\ Abdelaziz Omari ${ }^{\mathrm{d}}$ \\ ${ }^{a}$ Arts et Métiers ParisTech, Rue Saint Dominique, 51006 Châlons-en-Champagne, France \\ ${ }^{\mathrm{b}}$ Department of Chemical Engineering, University of Waterloo, 200 University Avenue, West Waterloo N2L 3G1, ON, Canada \\ ${ }^{\mathrm{c}}$ I2M, Arts et Métiers Paris-Tech, CNRS, Esplanade des Arts et Métiers, 33405 Talence Cedex, France \\ d I2M, Bordeaux-INP, CNRS, Esplanade des Arts et Métiers, 33405 Talence Cedex, France
}

\begin{abstract}
Mercury Intrusion Porosimetry (MIP) is still today the reference porosimetry technique despite its environmental health and safety concerns. As a safe alternative, the Yield Stress fluids Method (YSM) consists in computing the Pore Size Distribution (PSD) of a given material from the pressure drop vs. flow rate measurements during injection of a yield stress fluid. However, the question arises whether the PSDs provided by YSM are representative of the actual pore dimensions. To answer this question, three numerical methods to obtain the PSD from digital images are proposed and compared in the present work. First, direct numerical simulations of YSM tests are performed in the considered media. Then, realistic PSDs are extracted from the images by using pore Network Modelling (NM). Furthermore, the obtained networks are also used to simulate MIP tests. The quantitative numerical results allow the evaluation of the relevance of YSM as an alternative to toxic MIP.
\end{abstract}

\section{Introduction}

Underground fluid transport processes and mechanical properties of porous media are intimately related to microscopic features of the pore structure, and especially to the Pore Size Distribution (PSD). Among others, notable examples are the moisture retention curve (Roel et al., 2001), the filtration capacity to remove contaminants (Suresh Kumar et al. , 2019), the permeability (Minagawa et al., 2008; Zhang et al., 2016), the uniaxial compressive strength (Wu et al., 2018) and the motion of the meniscus in multiphase flow (Payatakes and Dias, 1984). Therefore, proper characterization of PSD is crucial in a great number of industrial applications, e.g. in situ remediation of contaminated groundwater, separation processes, transport of landfill leachates, oil and gas industry, $\mathrm{CO}_{2}$ sequestration, transport of seawater through underground aquifers or geothermal energy generation.

At the present time, Mercury Intrusion Porosimetry (MIP) is the reference experimental method to obtain the PSD of dry samples of porous media (Van Brakel, 1981; León and León, 1998; Giesche, 2006., Minagawa et al., 2008; Muller and Scrivener, 2017;

\footnotetext{
* Corresponding author.

E-mail address: antonio.rodriguezdecastro@ensam.eu (A. Rodríguez de Castro).
}

Salejova et al., 2011; Cieszko et al., 2019). Some substantial benefits of this extensively used technique include the relatively fast measurements, the possibility of covering a large range of pore sizes, i.e., from $10^{-9}$ up to $10^{-3} \mathrm{~m}$ (Zhang et al., 2016) and the richness of accessible databases. Such advantages make the replacement of MIP challenging, in spite of the recent restrictions on the use of mercury as penetrant fluid introduced by Minamata Convention (United Nations, 2013) and the urgent appeal of the international scientific community for the development of clean alternatives (Rouquerol et al., 2012). Moreover, attention must be drawn to the serious shortcomings of MIP. In this regard, MIP is not convenient to characterize materials having important ink-bottle effects, due to overestimation of the volume of small pores and the underestimation of the volume of large pores (Roel et al., 2001; Salejova et al., 2011). Besides, the standard version of this method is limited to dry and consolidated samples at room temperature (Minagawa et al., 2008). Also, the high external pressures applied to the samples during the measurements can damage the pore network to be characterized (Li et al., 2018). Several researchers have simulated MIP tests based on percolation theory and multiphase flow models. For example, Tsakiroglou and Payatakes (2000) used simulations of MIP combined with the results of serial tomography to estimate the throat-diameter distribution of Vosges sand- 
stone. Furthermore, Hyväluoma et al. (2004) performed LatticeBolzmann simulations of MIP, reporting the considerable computational power demanded due to the high number of required iterations. In this regard, the rapid development of high-performance computers and the geometrical simplifications introduced by the use of pore network models can significantly reduce the computation time, especially in 3D porous media.

$\mathrm{X}$-ray micro-computed tomography is widely used to characterize the microscopic features of 3D porous media (Lindquist and Venkatarangan, 2000; Burlion et al., 2006; Caulkin et al., 2014; Houston et al., 2017; Saxena et al., 2018). In particular, the digital images acquired with this technique provide input geometries to numerical simulations of fluid flow. Permeability, tortuosity and other relevant quantities can be subsequently computed by using Lattice-Boltzmann or Computational Fluid Dynamics approaches. Recently, Bultreys et al. (2016) have comprehensively reviewed the existing imaging techniques for geological porous media as well as the direct simulation and pore Network Modelling approaches for the computation of transport properties. A major advantage of this method, which has been considered by some authors as a "gold standard" (Arns, 2004), is the non-destructive and the non-invasive nature of the tests (Caulkin et al., 2014). Nonetheless, the experimental equipment is costly, and, despite recent progress, spatial resolution $(\sim 1 \mu \mathrm{m})$ still represents a strong limitation of this method given that the throats of a size smaller than the lowest limit of resolution cannot be measured or are assigned to larger throat sizes. Another central issue arises from the reassignment of voxels along the pore-solid interface and the subsequent change in pore connectivity depending on voxel size (Guan et al., 2019). Also, the thinness of the used samples leads to poor representativeness of some geological samples (Bultreys et al., 2016). Moreover, the quality of the input image, the choice of filter during postprocessing steps and the segmentation method may induce significant changes in the obtained binary pore and solid networks leading to user-dependent results (Sheppard et al., 2004; Guan et al., 2019). In this respect, Guan et al. (2019) implemented a method for estimating errors in the calculations of porosity and permeability from X-ray tomographic images.

Other techniques are also available for the determination of PSD, including nitrogen adsorption method, water desorption porosimetry (Rouquerol et al., 2012; Bultreys et al., 2016), and NMR methods (Coates et al., 1999; Arns, 2004). Nevertheless, the preparation of the samples is highly elaborated in the adsorption methods and proper interpretation of the NMR measurements require accurate knowledge of the properties of both the skeleton and the fluid in the pore space (Cieszko et al., 2019). Also, the use of a set of different porosimetry techniques is the appropriate means to characterize the PSDs of porous media having an extremely wide range of pore sizes (Bultreys et al., 2016), such as tight reservoir rocks (Wang et al., 2018), porous building materials (Roel et al., 2001), some types of sandstones (Tsakiroglou and Payatakes, 2000) and shales (Chen et al., 2018; Alfi et al., 2019). In this regard, MIP data sets along with SEM images of reservoir rocks and mineral soils were combined with percolation-type approaches and inverse modeling algorithms to characterize the pore structure of heterogeneous materials with a broad range of pore length scales, spanning some orders of magnitude (Tsakiroglou and Ioannidis, 2008; Tsakiroglou et al., 2009; Amirtharaj et al., 2011).

In the past, Ambari et al. (1990) proved that, in theory, the PSD of a bundle of parallel, non-intersecting cylindrical tubes of equal length but of different radii can be derived from the measurement of the flow rate $\mathrm{Q}$ as a function of pressure gradients $\nabla \mathrm{P}$ during the injection of a yield stress fluid. For this purpose, analytical and numerical methods requiring complex techniques of polynomial filtering were proposed in previous researches in order to achieve inversion of $\mathrm{Q}$ vs. $\nabla \mathrm{P}$ datasets obtained from numeri- cal (Oukhlef, 2011; Oukhlef et al., 2014) and laboratory (Malvault, 2013) experiments. The Yield Stress porosimetry Method (YSM) was developed taking advantage of the preceding investigations towards an industry-oriented method which was directly applicable to raw experimental measurements without any filtering or interpolation (Rodríguez de Castro et al., 2014). The inversion algorithm consists in isolating the increase in flow rate arising from yielding of the fluid in the pores of a given size, i.e., smaller pores as the the applied pressure gradient is gradually increased. In the search for a suitable penetrant fluid with negligible degrees of viscoelasticity and thixotropy, xanthan gum aqueous solutions were proposed by Rodríguez de Castro et al. (2014), and the polymer concentration was optimized in order to obtain accurate PSDs (Rodríguez de Castro et al., 2018). Furthermore, the critical experimental issues of mechanical degradation, retention and adsorption of xanthan macromolecules were also assessed in previous research (Rodríguez de Castro et al., 2016). The possibility of obtaining the signature of PSD from non-Newtonian fluid flow in porous media was subsequently explored by other authors, which proposed a similar porosimetry method (Abou Najm and Atallah, 2016; Atallah and Abou Najm, 2019).

The amount of available research work addressing numerical simulation of yield stress fluids flow in porous media is rather limited, the major stumbling block being the considerable computation times required to solve strong non-linearities. It must be mentioned here that the non-linearities in the transport equations arise from the viscosity models of the considered non-Newtonian fluids only. Indeed, the investigated flows are generally considered within the creeping regime and no inertial (non-linear) term (Agnaou et al., 2017) is used to model the fluid flow. Pore Network Modelling was used by Balhoff et al. (2012) to simulate the flow of complex fluids through packs of spheres and sandstones, with the objective of minimising the computation time. Regarding direct numerical simulations, Ouyang et al. (2013) used a 3D finite volume method to evaluate the impact of yield stress on the value of the pressure gradient generated under a given injection velocity during flow through a single 3D micro-channel within a cubic packing of hard spheres. In the same work, the streamlines, the pressure contours and the velocity fields were displayed, showing the variations in shape and dimensions of the central unyielded zone for different values of the rheological parameters. Other authors have performed direct numerical simulations in order to assess the dependence of apparent viscosity on injection velocity for non-yielding complex fluids by using digital images of real rocks, e.g., 2D images provided by Scanning Electronic Microscopy (Tosco et al., 2013) or X-ray microtomography images (Zamani et al., 2017). Moreover, the single- and two-phase flows of shear-thinning fluids in porous media were theoretically analysed in previous works with volume averaging, pore network simulations, and critical path analysis, and the so-produced models have been validated with experiments in model porous media (Shah and Yortsos, 1995; Tsakiroglou, 2002b; 2004).

Chevalier and Talon (2015) reported, from the results of numerical experiments, the progressive opening of flow paths as the applied pressure gradient is increased during yield stress fluids flow through a 2D porous medium. Furthermore, the effects of varying cross section of different shape in the bundle of capillaries model on the relationship between flow rate and pressure gradient were investigated by Malvault et al. (2017) by means of numerical simulations at the pore scale. These authors showed that varying the size of the cross section along the axial direction induces important changes in the threshold pressure gradient as compared to capillaries with uniform section. Also, Bao et al. (2017) conducted numerical simulations of yield stress fluids with constant plastic viscosity (Bingham fluids) through rough walled-fractures, in order to predict the effects of varying pressure gradient on flow rate and 
channelling. In a recent review, Saramito and Wachs (2017) analyzed and compared the existing approaches to simulate viscoplastic fluid flows, highlighting the need for large supercomputers in 3D numerical simulations of yield stress fluids flow. These machines should be available in the near future. It should be noted that, despite the encouraging results recently presented in the literature demonstrating the feasibility of YSM as a potential alternative porosimetry technique, in none of the previous works the flow patterns generated during the injection of a yield stress fluid through a well-characterized porous medium were analysed in detail. This caused confusion regarding the physical meaning of the characterized pore dimensions.

The aim of the present paper is to assess the efficiency of three different methods allowing the computation of PSD from a segmented image of the investigated porous media: simulated YSM, simulated MIP and direct determination from pore network models of these porous media. To do so, a set of numerical flow experiments with three different 2D porous media are conducted in which the flow rate generated at various pressure gradients during the injection of a yield stress fluid are computed in order to obtain the input raw data to serve later for YSM. The computed YSM-PSD is subsequently compared to the pore body and throat size distributions of the pore networks extracted from digital images of the media. Also, a MIP test is numerically simulated by using the pore network as input geometry in order to obtain the PSDs provided by this reference technique. Special attention will be paid to the numerical validation of YSM as a satisfactory non-toxic porosimetry technique and the accuracy of the obtained PSDs. In particular, the physical meaning of the pore dimensions obtained from YSM will be elucidated through careful inspection of the shear viscosity maps and the pressure contours deduced from the numerical simulations.

\section{Numerical experiments: extracting Pore Size Distribution from digital images}

\subsection{Porous media under consideration}

Three different two-dimensional (2D) porous structures were considered in this study. The first one, shown on Fig. 1(a), has a porosity of 0.35 and consists of a model porous structure of a medium made of disordered parallel circular cylinders of identical diameters. The generation of this structure was performed considering first a uniform disc packing in a triangular tiling by using a constant disc diameter. Then, the position of the circular cylinders was randomly varied within the uniform geometrical tiling units, assuming a uniform distribution. In addition, in order to avoid computational grid generation issues, a minimum distance of $3 \%$ of the cylinders' diameter between neighbour cylinders was imposed. This ensures a geometry with no contact between the cylinders. The second porous medium, was a poorly sorted unconsolidated fluvial sand pack from southern Australia with a porosity of $36.3 \%$, which was imaged using an X-ray micro-CT device by Sheppard and Prodanovic (2015) and is freely available. The 2D slice of the medium displayed in Fig. 1(b) was used in the present investigation. Finally, the third medium is a sandy porous structure with a porosity of $38.9 \%$, (see Fig. 1(c)). This medium was obtained from slicing an open-source dataset containing a series of synchrotron X-ray microtomography images of a sandy quartz column (Molnar, 2016). It can be observed that the solid grains are sharper in the sand pack than in the sandy quartz.

\subsection{Numerical experiments with Yield Stress fluids Method}

The standard YSM algorithm allowing the computation of PSD from a set of $(\nabla \mathbf{P}, Q)$ data obtained during the flow of a yield

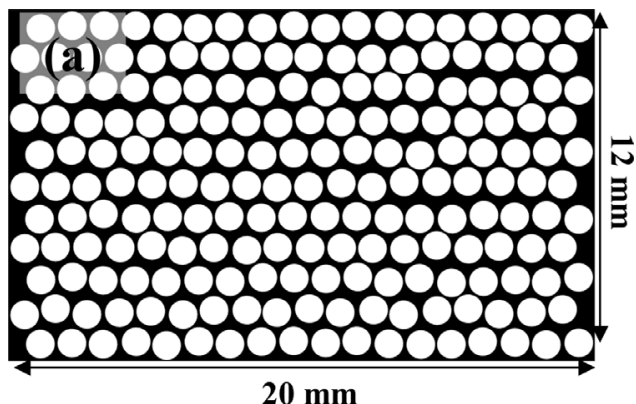

(b)

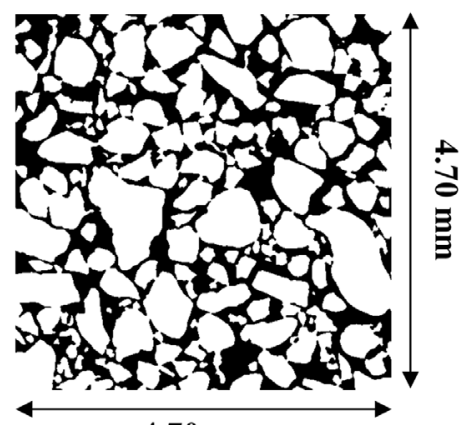

$4.70 \mathrm{~mm}$

(c)

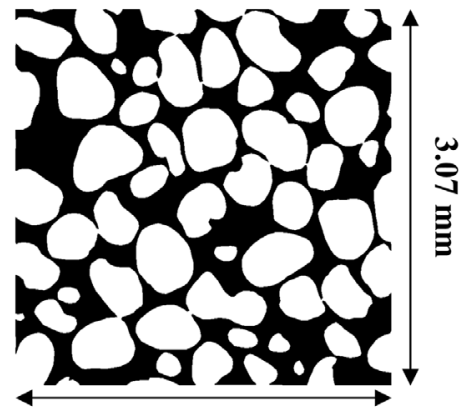

$3.07 \mathrm{~mm}$

Fig. 1. Geometry and dimensions of the three investigated 2D porous media: (a) disordered matrix of circular solid cylinders (b) slice of sand pack (Sheppard and Prodanovic, 2015) and (c) slice of sandy quartz medium (Molnar 2016).

stress fluid through the characterized medium was presented and validated in previous works (Rodríguez de Castro et al., 2014, 2016, 2018). Hence, the current subsection will only address the minor modifications that are needed in order to adapt the method to 2D porous media, as well as the central features of YSM.

The shear-flow rheological behaviour of common yield stress fluids under steady-state conditions is modelled in YSM by means of Herschel-Bulkley's empirical law (Herschel and Bulkley, 1926), which can be expressed as follows:

$\left\{\begin{array}{l}\tau=\tau_{0}+\mathrm{k} \dot{\gamma}^{\mathrm{n}} \text { for } \tau \geq \tau_{0} \\ \dot{\gamma}=0 \text { for } \tau \leq \tau_{0}\end{array}\right.$

with $\tau$ being the shear stress at a given shear rate $\dot{\gamma}, \tau_{0}$ the yield stress of the fluid, $\mathrm{k}$ its consistency and $\mathrm{n}$ its flow index. $\tau_{0}$, $\mathrm{k}$ and $\mathrm{n}$ are commonly obtained by fitting the $(\tau, \dot{\gamma})$ data measured with a rheometer. $\tau_{0}=10 \mathrm{~Pa}, \mathrm{k}=1 \mathrm{~Pa} \mathrm{~s}$ and $n=0.5$ were used for the injected fluid in the present simulations.

YSM is based on the following assumptions:

- 1. Incompressible flow.

- 2. Creeping regime (inertial pressure losses are negligible).

- 3. Wall-slip is negligible.

- 4. The rheological properties of the injected fluid and the dimensions of the pores remain unchanged during its flow through the porous medium. In the case of the polymer solu- 
tions injected in real laboratory tests (Rodríguez de Castro et al., 2014, 2016, 2018), the mechanical degradation, retention and adsorption of the macromolecules is neglected. This assumption was shown to be realistic in the case of highly permeable media (Rodríguez de Castro et al., 2016).

- 5. In the 3D case, YSM assumes that the dimensions controlling macroscopic pressure losses when injecting a fluid through a homogenous and isotropic porous medium are well represented by pore radii of a bundle of parallel capillaries. Some modifications must be made when extending the method to $2 \mathrm{D}$ porous media. That is to say, a bundle of rectangular channels with varying aperture and infinite width in the direction perpendicular to flow. In this model, the frequencies of the different pore apertures hi are distributed according to a probability distribution $\mathrm{p}(\mathrm{hi})$.

The wall shear stress $\tau_{\mathrm{w}}$ under a given pressure gradient $\nabla \mathrm{P}$ for the steady flow of an incompressible fluid through a rectangular pore of constant aperture $h$ and infinite width (2D case) can be expressed as:

$\tau_{\mathrm{w}}=\frac{\nabla \mathrm{Ph}}{2}$

Therefore, flow through a channel of aperture $h$ will only occur when the threshold pressure gradient $\nabla \mathrm{P}_{0}=\frac{2 \tau_{0}}{\mathrm{~h}}$ is exceeded, and the total flow rate per unit depth through a bundle of rectangular capillaries can be computed by using the following expression:

$\mathrm{Q}(\nabla \mathrm{P})=\sum_{\mathrm{i}=1}^{\mathrm{N}} \mathrm{n}_{\mathrm{i}} \mathrm{q}\left(\nabla \mathrm{P}, \mathrm{h}_{\mathrm{i}}\right)$

with $\mathrm{q}\left(\nabla \mathrm{P}, \mathrm{h}_{\mathrm{i}}\right)$ being the volumetric flow rate per unit depth of a Herschel-Bulkley fluid through a rectangular channel of aperture $h$ and infinite width as a function of $\nabla$ P, as given by Skelland (1967), and more recently by Ferrás et al. (2012). During a standard YSM laboratory experiment, a set of $\mathrm{N}$ flow rates are imposed and the steady state pressure gradients $\nabla \mathrm{P}_{\mathrm{i}}(\mathrm{i}=1 \ldots \mathrm{N})$ are measured. Analogously, $\nabla \mathrm{P}_{\mathrm{i}}$ is imposed during the numerical YSM simulations performed in this work and the resulting flow rate per unit depth $\left(\mathrm{m}^{2} / \mathrm{s}\right) \mathrm{Q}_{\mathrm{i}}$ is computed, obtaining a set of $\mathrm{N}\left(\nabla \mathrm{P}_{\mathrm{i}}, \mathrm{Q}_{\mathrm{i}}\right)$ data. The procedure followed during these simulations is presented below.

The flow problems considered in this work were numerically solved using the finite-element-method-based simulation package, Comsol Multiphysics version 5.3. (2017). The porous media considered in this study (Fig. 1) were discretized using unstructured triangular meshes. The simulations were carried out using the Creeping Flow module, developed for solving Stokes flow problems. The boundary conditions associated with the flow problem consist of the Dirichlet uniform pressure at the left and right boundaries of the porous structures. In addition, a no-slip velocity boundary condition was imposed at the solid-fluid interface as well as at the top and the bottom boundaries of the considered porous media. It must be noted that since flow is induced thanks to the enforced pressure difference, backflow may appear at the inlet and result in numerical instabilities. For this reason, the backflow at the entrance (left boundary) was suppressed. This constraint can be compared to the situation where one uses a pump to inject the fluid through the porous medium and where the pump does not allow the fluid to go back. To overcome the expected singularities when using a true yield stress fluid relationship, the viscosity of the fluid was described as follows:

$\mu=\min \left[\mu_{\max },\left(\mathrm{k} \dot{\gamma}^{\mathrm{n}-1}+\frac{\tau_{0}}{\dot{\gamma}}\right)\right]$

in which $\mu_{\max }$ is the maximum viscosity. A value $\mu_{\max }=$ 10000 Pa s was used in this study. It should be highlighted, how-

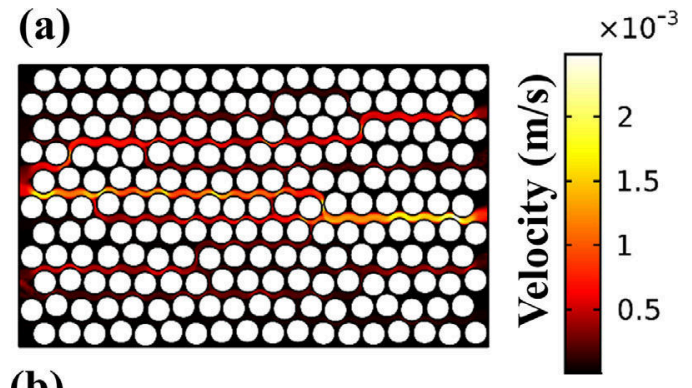

(b)

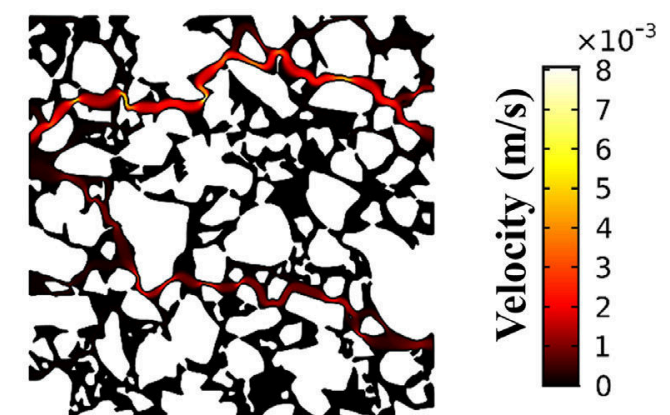

(c)

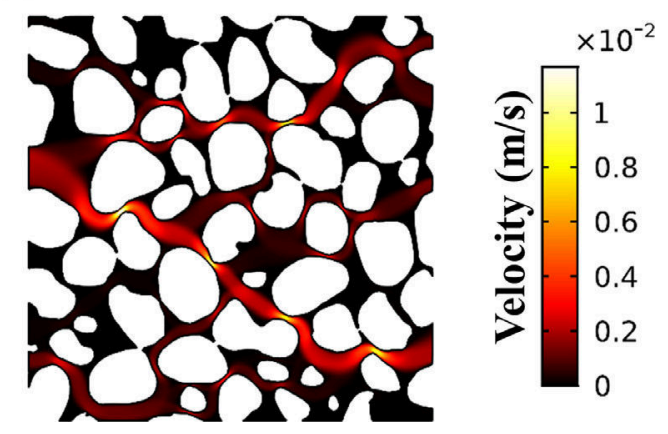

Fig. 2. Examples of the velocity magnitude maps provided by simulations in the three porous media: (a) disordered matrix of circular solid cylinders (b) sand pack and (c) sandy quartz medium.

ever, that this maximum limit was adopted as a compromise between accuracy and numerical stability. In fact, using extremely high $\mu_{\max }$ values yields important viscosity gradients within the computational domain and numerical instabilities. On the other hand, low $\mu_{\max }$ values fail to reproduce the expected rheological behaviour. The resulting non-linear system of equations was solved using the Comsol Stationary Solver and the solution was sought using the Newton-Raphson algorithm, taking as initial guesses the initial conditions (fluid at rest, zero pressure and velocity fields). The numerical solution is then considered converged upon reaching a residual below a relative tolerance of $10^{-3}$. The system of linearized equations within each Newton-Raphson iteration, was solved using the direct solver PARDISO (Schenk and Gärtner 2004). In Fig. 2, examples of the fluid velocity magnitude maps at intermediate pressure gradients are displayed over the three considered porous structures showing the existence of an important channelling effect through the largest pores. Such channelling effect was interpreted with reference to the critical path analysis of percolation theory in the literature (Hunt, 2001; Hunt and Gee, 2002; Tsakiroglou, 2002a), applied to the case of power-law fluids (Shah and Yortsos, 1995).

For the numerical experiments in a given 2D porous medium, a group of $\mathrm{N}$ representative aperture classes is formed by $\mathrm{h}_{\mathrm{i}}=$ $\alpha \frac{2 \tau_{0}}{\nabla \mathrm{P}_{\mathrm{i}}}\left(\mathrm{h}_{1}>\mathrm{h}_{2}>\cdots>\mathrm{h}_{\mathrm{N}}\right)$, where $\alpha$ is a numerical factor strictly 
greater than unity. This condition is due to the fact that, under a given pressure gradient $\nabla \mathrm{P}_{\mathrm{j}}$, the fluid remains unyielded in a pore unless its aperture is strictly greater than $\frac{2 \tau_{0}}{\nabla P_{\mathrm{j}}}$. The value $\alpha$ can be determined by adopting the standard procedure which was comprehensively explained in preceding works (Rodríguez de Castro et al., 2014, 2016). The number of pores $n_{j}$ of a particular aperture class $h_{j}$ is calculated as being the maximum positive value respecting the condition $\mathrm{Q}_{\mathrm{s}} \geq \sum_{\mathrm{g}=1}^{\mathrm{j}} \mathrm{n}_{\mathrm{g}} \mathrm{q}\left(\nabla \mathrm{P}_{\mathrm{s}}, \mathrm{h}_{\mathrm{g}}\right)$ for every value of $s \geq j+1$. The number of pores of the different aperture classes are computed starting from the largest pores and decreasing pore size, i.e., from $n_{1}$ to $n_{N}$. Then, the relative frequency of each pore aperture class $h_{j}$ is calculated as $p\left(h_{j}\right)=\frac{n_{j}}{\sum_{i=1}^{N} n_{i}}$, yielding the PSD of the medium.

\subsection{Extraction of pore networks}

Pore network models are used as an idealized representation of a real pore space in order to simplify the procedure for solving transport problems. These models are considered more realistic than the traditional bundle-of-capillaries. Pore networks allow for numerical simulations with relatively low computational costs compared to the continuum approach using numerical methods such as the lattice Boltzmann or finite element method. In addition, since simulations based on the pore Network Modelling only require limited computational resources, one can consider large porous medium samples for investigation. This prevents from the need to extract a Representative Elementary Volume (REV), which is not always easy as the REV's size was shown to vary with the Reynolds number even on ordered structures (Agnaou, 2016). Moreover, essential features for permeability and pressure loss prediction, such as throat constriction ratio and pore connectivity, can be well characterized by the pore network models in typical cases (Paul et al., 2019). A review of the advances in the pore Network Modelling approach of porous media was presented by Xiong et al. (2016), in which the procedure adopted in this work to build pore networks, and current applications ranging from dissolution phenomena to biomass growth are detailed.

Pore network extraction operations carried-out in this work were performed using the subnetwork of the oversegmented watershed (SNOW) algorithm (Gostick, 2017) implemented within the open-source toolkit for quantitative analysis of porous media images, PoreSpy (Gostick et al., 2019). This algorithm can be applied to different types of porous media, which makes it a good choice for this work. The PoreSpy package also includes an algorithm allowing simultaneous extraction of void and solid phase networks from tomographic images (Khan et al. 2019), with applications in catalytic reactors and electrochemical systems. Furthermore, the SNOW algorithm was implemented in Python which makes it easily extensible. It also relies on the Scipy stack and allows for a relatively short extraction (network generation) time. The algorithm proceeds through different steps (Gostick, 2017) where it first extracts the distance map of the void spaces, filters it and eliminates peaks on saddles and plateaus. It then merges peaks that are close to each other. Finally, it assigns void voxels to pores. The SNOW algorithm was used for the extraction of the pore networks from the $2 \mathrm{D}$ medium made of circular cylinders, the sand pack and the sandy porous structure shown on Fig. 1.

\subsection{Mercury intrusion porosimetry experiments}

After extracting the pore networks using the SNOW algorithm, MIP simulations were performed making use of the open-source pore Network Modelling package OpenPNM (Gostick, 2016). For this, the pore networks files, containing the network and all the geometrical information, were first imported using an OpenPNM script. Then, the OpenPNM MIP algorithm was used to perform the simulations. This algorithm is based on the Washburn equation as described in what follows.

Washburn equation (Washburn, 1921) is commonly used in the traditional version of MIP to relate the applied capillary pressure to the invaded pore dimension (Good and Mikhail, 1981; Giesche, 2006; Cieszko et al., 2019). In the case of 2D porous media, this equation can be written as follows:

$\mathrm{P}_{\mathrm{ci}}=-\frac{2 \sigma \cos \theta}{\mathrm{h}_{\mathrm{i}}}$

with $h_{i}$ being the aperture of the pore. Simulations were performed by assuming a contact angle $\theta=140^{\circ}$ and a surface tension of the mercury-air pair $\sigma=0.48 \mathrm{Nm}^{-1}$, which are routinely used values and are accepted in most practical situations (Giesche, 2006). In the preceding equation, $\mathrm{P}_{\mathrm{C}_{\mathrm{i}}}$ is the external pressure applied to mercury. It should be noted that the PSD provided by MIP is volume-weighted, as it defines the volume fraction of pores that are accessible through pore openings of a given size (Cieszko et al., 2019). For each 2D porous medium, simulations are performed over a wide range of pressure values. The resulting invading phase (mercury) saturation curve is then used to estimate the volume fraction distribution of the pore openings with Eq. (7).

\section{Results}

\subsection{Pore size distribution obtained by network modelling}

The procedure presented in Section 2.3. was applied to the three segmented images of Fig. 1, allowing the extraction of the pore networks displayed in Fig. 3. Since the images used in this study are two-dimensional, pores and throats of the extracted networks are assigned circular and rectangular shapes, respectively. The Body Size Distributions (BSDs) and Throat Size Distributions (TSDs) obtained for the three porous media are also shown in the same figure. It is first noted that all the TSDs are right-skewed, with the peak throat size being smaller than the average size in all media. The average body size of the matrix of circular cylinders is $519 \mu \mathrm{m}$. The BSD and the TSD do not overlap in this porous structure, which is in contrast with the sand pack and the sandy quartz media. The largest pores are situated in the left and right ends of the matrix of circular cylinders, while the rest of the medium is occupied by smaller pores with sizes ranging from $\sim 300 \mu \mathrm{m}$ to $\sim 700 \mu \mathrm{m}$ and a single probability peak at $525 \mu \mathrm{m}$. On the other hand, the TSD ranges from $\sim 20 \mu \mathrm{m}$ to $\sim 380 \mu \mathrm{m}$, with an average throat size of $126 \mu \mathrm{m}$ and a peak size of $54 \mu \mathrm{m}$. In the case of the sand pack, the average body and throat sizes are $174 \mu \mathrm{m}$ and $44 \mu \mathrm{m}$, respectively, and the shapes of both BSD and TSD are quite similar to those of the matrix of circular cylinders. As for the sandy quartz medium, the average body and throat sizes are $192 \mu \mathrm{m}$ and $71 \mu \mathrm{m}$, respectively. It is highlighted that some small pore bodies of a similar size as the throats exist in this medium. Also, the peak of the TSD at $44 \mu \mathrm{m}$ has a higher probability than for the preceding media.

\subsection{Pore size distribution obtained by numerical MIP}

The simulated capillary pressure curves can be obtained by employing the method presented in Section 2.4. This method was applied to the three investigated media, giving the results shown in Fig. 4. Through a brief inspection of Fig. 4(a), it is noted that the matrix of circular cylinders produces a much steeper capillary pressure curve, which leads to a PSD with a high-probability peak at $140 \mu \mathrm{m}$. This may reflect the fact that all the biggest openings towards the pore bodies have similar sizes and the medium is 

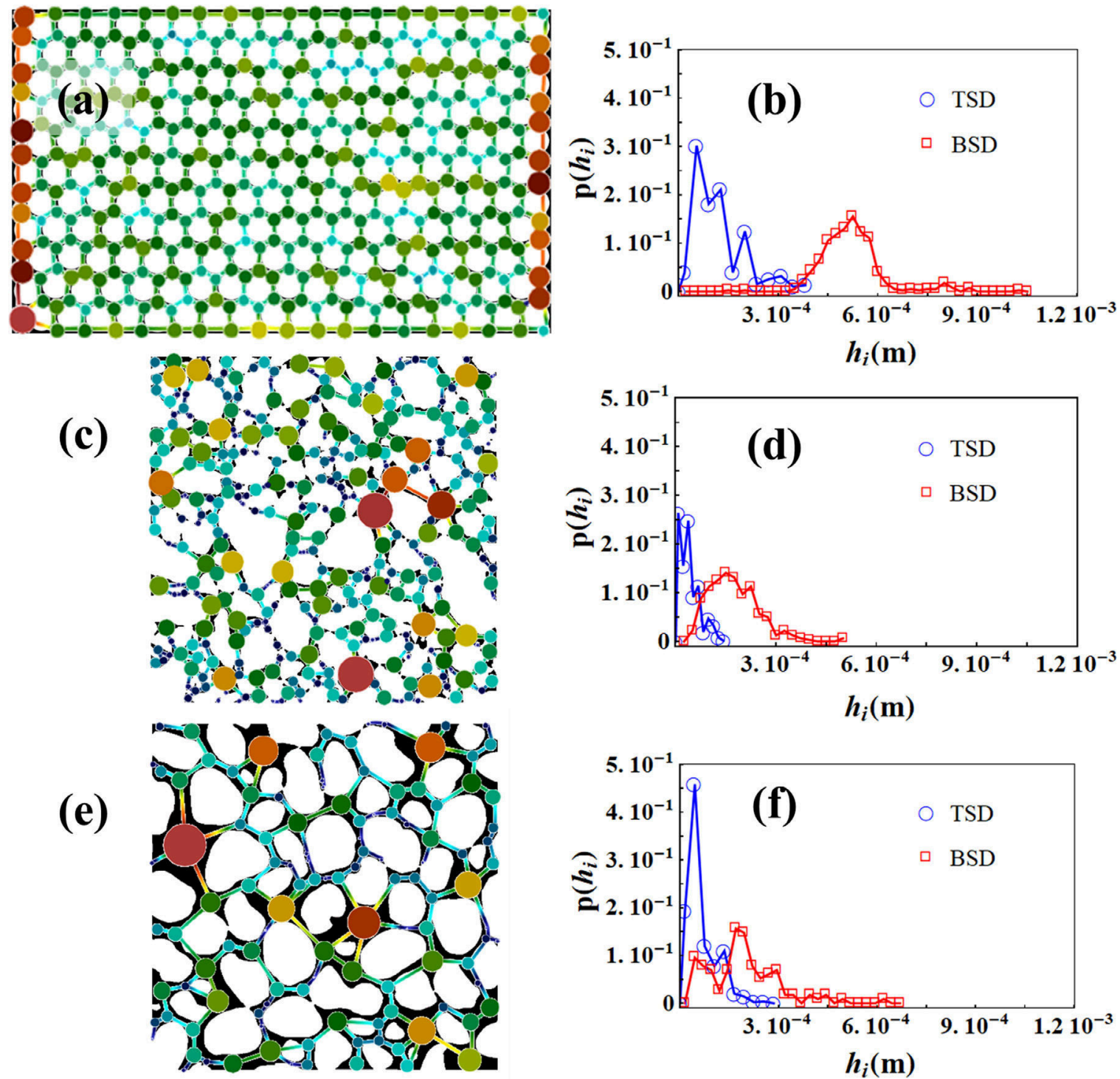

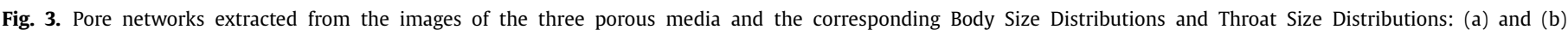

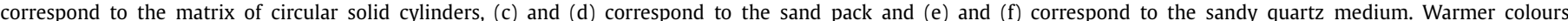
correspond to bigger dimensions in (a), (c) and (e).

nearly fully saturated once the threshold pressure of $\sim 5 \mathrm{~Pa}$ is exceeded. On the contrary, the capillary pressure curves of the other two media cover a wider range of pressures, and the saturation increases progressively as the pressure is increased. This may be the consequence of a more complex pore topology with pore openings of different sizes, as reflected by the overlapping PSDs and TSDs (Fig. 3). The higher level of pressure as compared to the matrix of circular cylinders was expected, given the smaller mean size of the pores. Moreover, despite the differences in terms of grain shape (Fig. 1), the PSDs of the sandy quartz and the sand pack are almost coincident, the main difference being the presence of some bigger pores in the sandy quartz.

\subsection{Pore size distribution obtained by yield stress fluid method}

As explained in Section 2.2., the ( $\nabla \mathbf{P}, \mathrm{Q})$ data resulting from the numerical injection of the yield stress fluid through the porous medium to be processed are used as an input to YSM algorithm. The simulated $(\nabla \mathbf{P}, Q)$ data for the three porous media are represented in Fig. 5(a), showing the non-linear relationship between $\nabla \mathbf{P}$ and $\mathrm{Q}$ arising from the shear-thinning behaviour and the extremely high viscosity values exhibited by the fluid at the lowest values of $\nabla \mathbf{P}$. Indeed, $\mathrm{Q}$ approaches zero below a threshold pressure gradient $\left(\sim 10^{5} \mathrm{~Pa} / \mathrm{m}\right.$ for the matrix of circular cylinders and $\sim 3 \times 10^{5} \mathrm{~Pa} / \mathrm{m}$ for the sand pack and the sandy quartz). It is obvious from the same figure, that the $\mathrm{Q}$ vs. $\mathrm{h}_{0}^{*}=2 \alpha \tau_{0} \frac{\mathrm{TL}}{\Delta \mathrm{P}_{0}}=\mathrm{Th}_{0}$ of the sand pack and the sandy quartz are remarkably similar, in spite of the differences in terms of grain morphology that can be observed in Fig. 1.

The PSDs of the three porous media were calculated from the raw $(\nabla \mathbf{P}$, Q) data, by applying the algorithm presented in Section 2.3, and the results are displayed in Fig. 5(b). It is observed that YSM is capable of discriminating the considerably bigger pores in the matrix of circular solid particles from the smaller pores in the sand pack and the sandy quartz medium. Moreover, the characterized pore sizes in the sand pack are slightly smaller than those in the quartz medium, which is a consequence of the slightly higher pressure gradients being generated at the lowest flow rates (Fig. 5(a)). It can be noticed that the PSD of the sand pack is bimodal, which might be a consequence of a higher vari- 

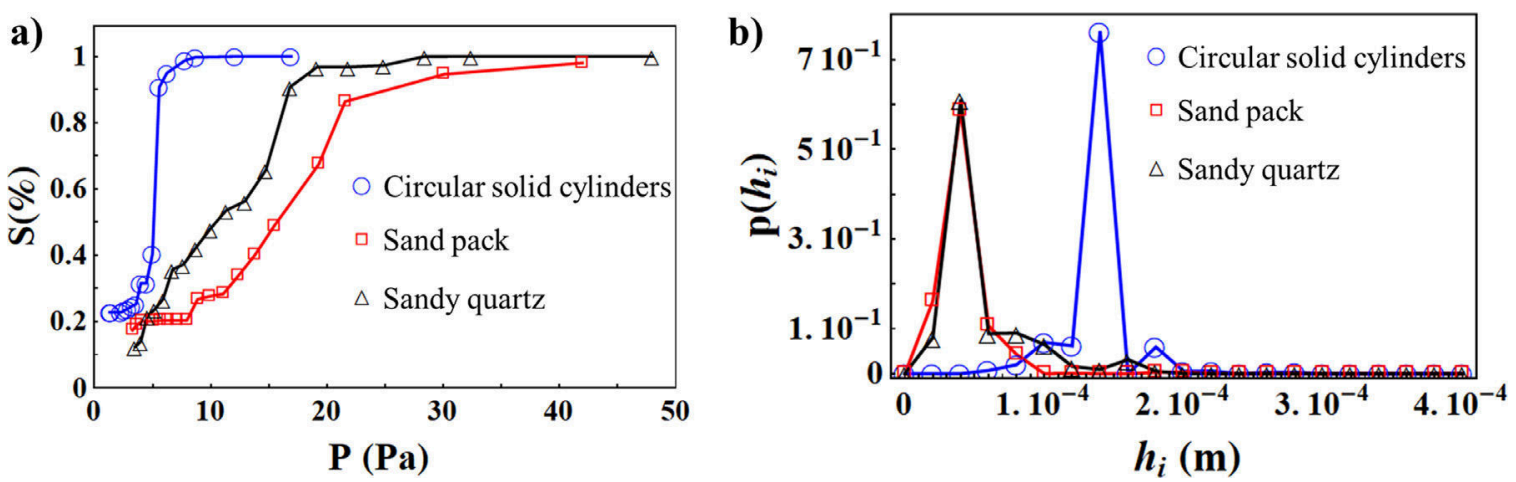

Fig. 4. Numerical MIP applied to the three porous media: (a) Simulated capillary pressure curves used as input to MIP and (b) obtained Pore Size Distributions.
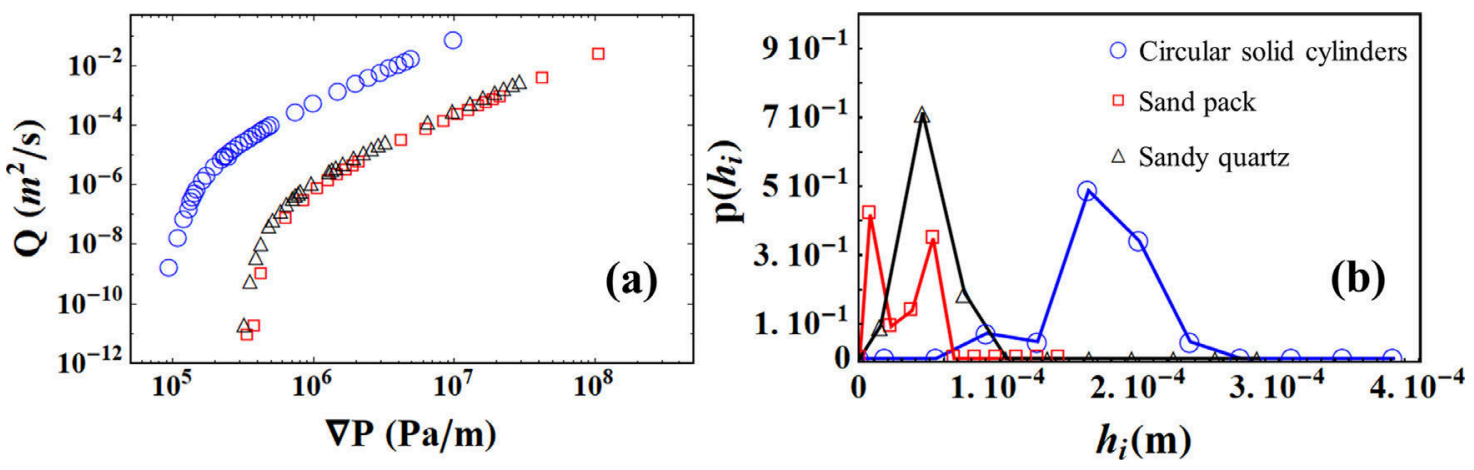

Fig. 5. Numerical YSM applied to the three porous media: (a) Simulated Q vs. $\nabla \mathbf{P}$ curves used as input to YSM and (b) obtained Pore Size Distributions.

ability in the local packing density due to the sharp-edged grains. Also, it is highlighted that the size resolution of the obtained PSDs is high enough to distinguish several pore size classes with significant probability in all the considered porous media.

\subsection{Comparison of the Pore Size Distributions obtained by the different methods}

In order to facilitate comparison between the PSDs obtained by each numerical porosimetry method, the results of the three adopted approaches are gathered together in Figs. 6 and 7. It should be noted that the characteristic length governing pressure drops is expected to be closer to the throat size than to the body size, which explains the good agreement between the TSD provided by Network modelling and the characteristic pore size distributions obtained with YSM and MIP (Fig. 6). In contrast, the body size is more useful to predict porosity and fluid saturation. An important result is the capability of all the three approaches to correctly reflect the order of decreasing pore sizes in the investigated media, i.e., the matrix of circular cylinders has the largest pores, followed by the sandy quartz, and the sand pack has the smallest ones for the three methods. Also, a noticeable feature of this comparison is that larger pore sizes are obtained with YSM in the simpler geometry of the matrix of circular cylinders. Indeed, YSM is expected to yield smaller pore sizes than the other approaches due to the higher pressure drops in the pore constrictions. However, in the particular case of the matrix of circular cylinders, preferential flow paths exist avoiding the smaller constrictions, as can be observed in Fig. 2(a), which may explain this striking result. On the contrary, YSM yields slightly smaller pore sizes in the more complex geometries of the sandy quartz and the sand pack. In these cases, the flow paths are more tortuous and go through some of the smallest constrictions.
It must be highlighted that there is a relatively good agreement between the PSDs obtained by means of the three methods (Fig. 6). In this regard, the range of pore sizes provided by these techniques is very similar and YSM reproduces the results of MIP with reasonable accuracy, although the two methods are based on distinct principles of measurement. Despite such general agreement, it is noted that the biggest pore throat sizes of the pore network have zero frequency in the PSDs of YSM for the quartz and the sand pack. This is attributed to their insignificant effect on Q vs. $\nabla \mathbf{P}$ relationships, as much lower pressure drops are produced in these big throats. More generally, it can be affirmed that the PSDs obtained by MIP and YSM are much closer to the TSDs (Fig. 6) than they are to the BSDs (Fig. 7) of the extracted pore networks, for the above-mentioned reasons. In an attempt to facilitate the proper assessment of the differences between the characteristic pore dimension distributions provided by the three methods, the means, standard deviations and probability peaks are listed in Table 1. Also, in order to estimate $\mathrm{K}$ from the pore size distributions provided by each method, it was first assumed that the mean of these distributions $m$ is equal to the hydraulic aperture $\bar{h}$ of the investigated medium. Then, the well-known equation $\overline{\mathrm{h}}=\sqrt{\frac{12 \mathrm{~K}}{\varepsilon}}$, obtained from combination of Hele-Shaw's law and Darcy's law was used to compute permeability. The obtained permeability values were subsequently compared to the one computed from DNS in Table 2 to assess the validity of the assumption. As expected, the estimation of $\mathrm{K}$ from the obtained PSDs is not always accurate, given that the characterized pore dimensions with the porosimetry methods are not close to the hydraulic aperture in all cases. In the particular case of the sand pack, the available correction factors proposed by Chauveteau et al. (1996) were applied to take into account the differences between the pore dimensions characterized by each method, resulting in improved accuracy of the prediction made from the results of YSM. 
Table 1

Mean (m), standard deviation ( $\mathrm{s}$ ) and probability maximum class (p) of the characteristic pore dimension distributions obtained by Network Modelling (TSD), MIP and YSM for the three porous media. Sample 1 (S1): matrix of circular solid cylinders; Sample 2 (S2): 2D slice of sand pack; Sample 3 (S3): 2D slice of sandy quartz medium.

\begin{tabular}{llllllllll}
\hline & $\mathrm{mYSM}(\mu \mathrm{m})$ & $\mathrm{m} \mathrm{MIP}(\mu \mathrm{m})$ & $\mathrm{M} \mathrm{NM}(\mu \mathrm{m})$ & $\mathrm{s}$ YSM $(\mu \mathrm{m})$ & $\mathrm{s}$ MIP $(\mu \mathrm{m})$ & $\mathrm{s} \mathrm{NM}(\mu \mathrm{m})$ & $\mathrm{p}$ YSM $(\mu \mathrm{m})$ & $\mathrm{p} \mathrm{MIP}(\mu \mathrm{m})$ & $\mathrm{p} \mathrm{NM}(\mu \mathrm{m})$ \\
\hline S1 & 174.6 & 138.0 & 125.5 & 30.9 & 27.5 & 89.1 & 169.2 & 131.9 \\
S2 & 26.9 & 48.1 & 44.3 & 21.7 & 31.7 & 34.7 & 54.3 & 34.1 \\
S3 & 57.1 & 75.0 & 70.7 & 10.5 & 41.7 & 44.4 & 59.1 & 43.7 & 45.0 \\
\hline
\end{tabular}

Table 2

Comparison between the permeability values computed with the three numerical porosimetry methods (YSM, MIP and TSD from NM) and the permeability value provided by Direct Numerical Simulation (DNS). Sample 1 (S1): matrix of circular solid cylinders; Sample 2 (S2): 2D slice of sand pack; Sample 3 (S3): 2D slice of sandy quartz medium.

\begin{tabular}{lllll}
\hline & K from DNS $\left(\mathrm{m}^{2}\right)$ & K from YSM $\left(\mathrm{m}^{2}\right)$ & K from MIP $\left(\mathrm{m}^{2}\right)$ & K from NM $\left(\mathrm{m}^{2}\right)$ \\
\hline S1 & $7.88 \times 10^{-10}$ & $8.89 \times 10^{-10}$ & $5.55 \times 10^{-10}$ & $3.94 \times 10^{-10}$ \\
S2 & $1.63 \times 10^{-11}$ & $1.66 \times 10^{-11}$ & $3.67 \times 10^{-11}$ & $4.49 \times 10^{-11}$ \\
S3 & $2.60 \times 10^{-10}$ & $1.06 \times 10^{-10}$ & $1.82 \times 10^{-10}$ & $1.62 \times 10^{-10}$ \\
\hline
\end{tabular}
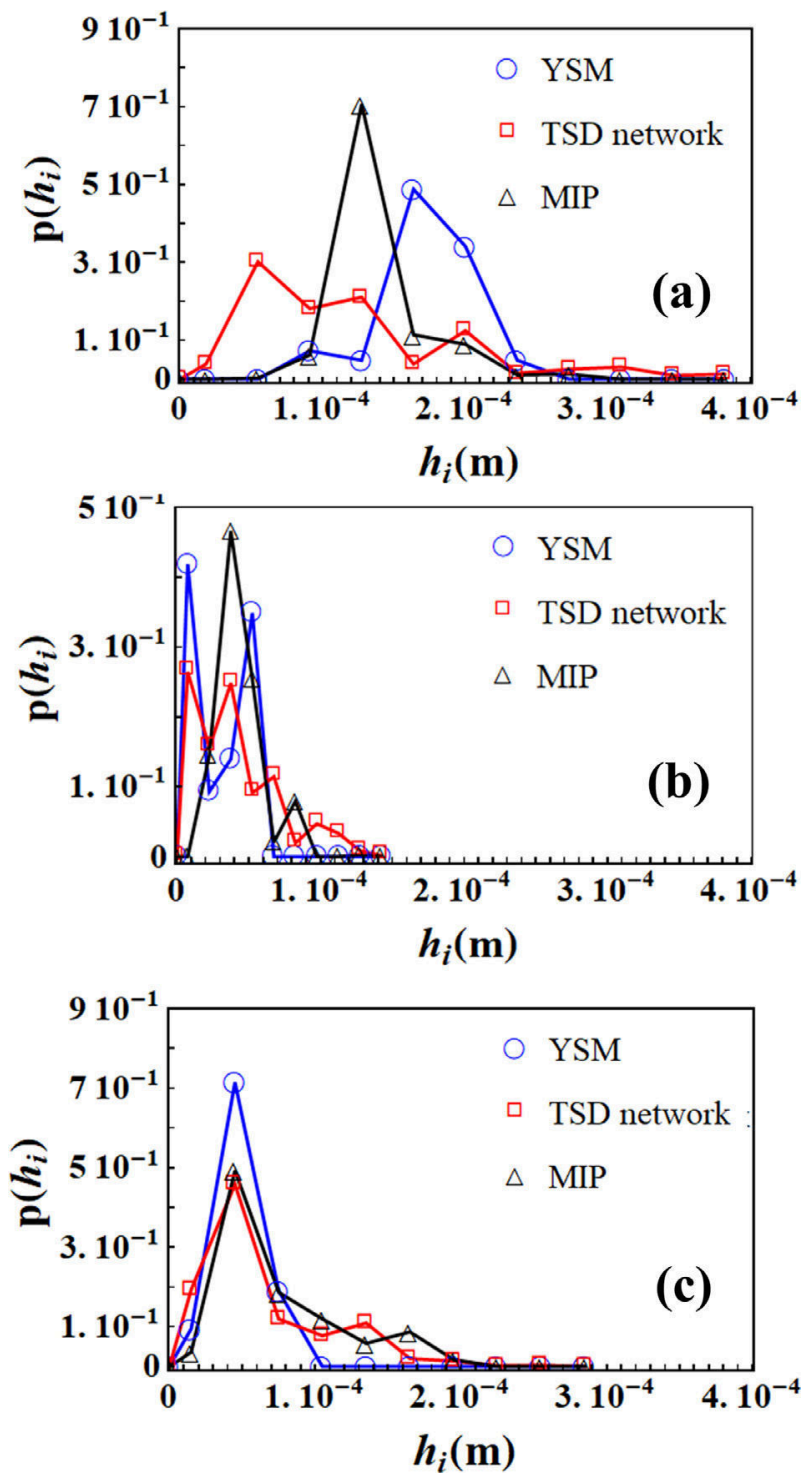

Fig. 6. Size distributions of characteristic pore dimensions provided by the three numerical porosimetry methods: (a) matrix of circular solid cylinders (b) 2D slice of sand pack and (c) 2D slice of sandy quartz medium.
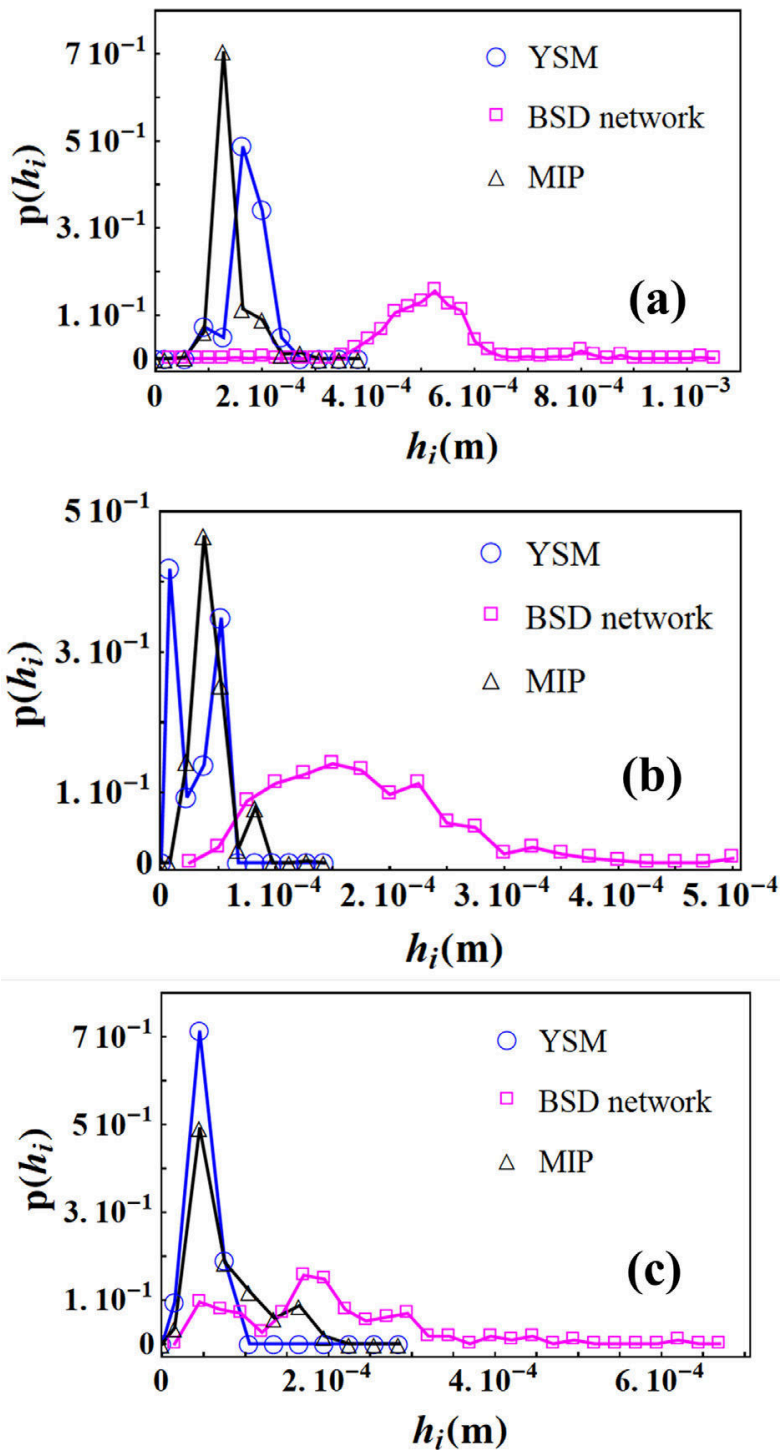

Fig. 7. Comparison between the BSD obtained with Network Modelling and the size distributions of characteristic pore dimensions provided by YSM and MIP: (a) matrix of circular solid cylinders (b) 2D slice of sand pack and (c) 2D slice of sandy quartz medium. 


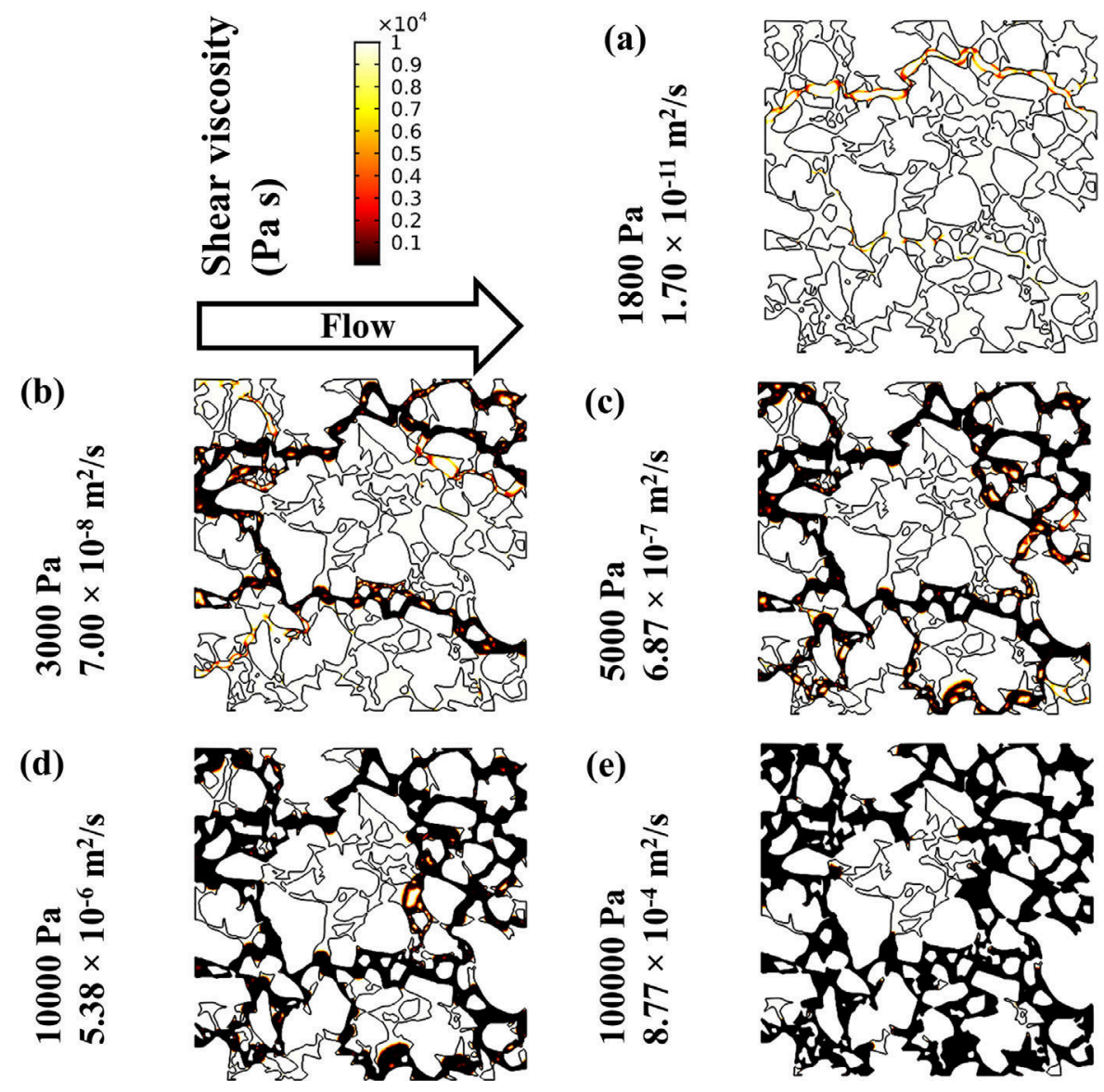

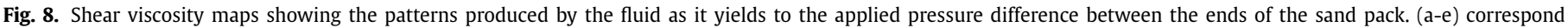
to different values of imposed pressure drops. Dark areas represent the regions in which the fluid flows.

\section{Discussion}

The pseudo-yield stress fluid model used in the numerical simulations presents a limiting value of shear viscosity at the lowest shear rates. This is in contrast to ideal yield stress fluids (e.g., Hershel-Bulkley model), for which the viscosity goes to infinity as the yield stress is approached. Furthermore, this is also the case of many real pseudo-stress fluids (Rodríguez de Castro et al., 2018), such as Carreau fluids with very high values of the plateau viscosities (lower Newtonian plateau). The preceding aspect may be seen as an important limitation of YSM. In order to carefully evaluate the impact of such approximation, the regions at which the fluid remains stagnant at each pressure drop were identified by exploiting the output of numerical simulations. As an illustration, the shear viscosity maps corresponding to the injection of the pseudo-yield stress fluid through the sand pack is represented in Fig. 8 for different values of the pressure drop. It can be deduced from these figures that the differences in terms of viscosity value between the stagnant and flowing regions are significant, resulting in roughly binary viscosity maps. Therefore, it can be concluded that the rheological model used in the present numerical simulations is capable of accurately representing the flow behaviour of a yield stress fluid. The used approach was similar to the one used by Prashant and Derksen (2011) to represent the constitutive equation of yield stress fluids with constant plastic viscosities (Bingham fluids). An analogous method to smooth the yield criterion is Papanastasiou's approximation (Papanastasiou, 1987), which was used in previous researches (e.g., Ouyang et al., 2013; Chevalier and Talon, 2015; Bao et al., 2017). By doing so, Chevalier and Talon (2015) conducted a numerical inves- tigation of path opening dynamics and analysed the size distribution of the unyielded clusters.

Of particular relevance is to gain further knowledge on the pore dimension being characterized by YSM. To achieve that goal, it is essential to identify the regions of the porous medium generating most pressure drop during the flow of the yield stress fluid, i.e., the smallest pore throats (Paul et al., 2019). It is reminded that the pressure drop is the measured quantity in a YSM test. With this objective in mind, a contour map representing different levels of pressure within the sand pack during the injection of the yield stress fluid under a pressure difference of $4000 \mathrm{~Pa}$ is displayed in Fig. 9. It should be noted that the stagnant zones are represented with white colour. In this figure, a sharp decrease in pressure corresponds to a transition between colours. Close observation of the image shows that such transitions are produced at the smallest pore constrictions, as expected. The average size of the pore constrictions in this image is $35 \mu \mathrm{m}$.

When defining the pore size class $\mathrm{h}_{0}=\alpha \frac{2 \tau_{0}}{\nabla \mathrm{P}_{0}}=2 \alpha \tau_{0} \frac{\mathrm{L}}{\Delta \mathrm{P}_{0}}$ that opens to the flow of the yield stress fluid under a pressure drop $\Delta P_{0}$, the algorithm of YSM assumes that the pores are straight and horizontal, with a length $L$ equal to the length of the bundle. In contrast, the length of a real channel with tortuosity $\mathrm{T}$ is given by $L^{*}=T \times L$, and the depth of the tortuous channel opening to the flow under the same pressure drop $\Delta \mathrm{P}_{0}$ is expressed as $\mathrm{h}_{0}^{*}=2 \alpha \tau_{0} \frac{\mathrm{TL}}{\Delta \mathrm{P}_{0}}=\mathrm{Th}_{0}$. The tortuosity factor $\mathrm{T}$ of the flow paths displayed Fig. 9 was estimated to be 1.31 , which was obtained by dividing the surface average of the velocity magnitudes by the surface average of the horizontal components of velocity over the pore space. If the average size of pore constrictions in Fig. 9 is divided 


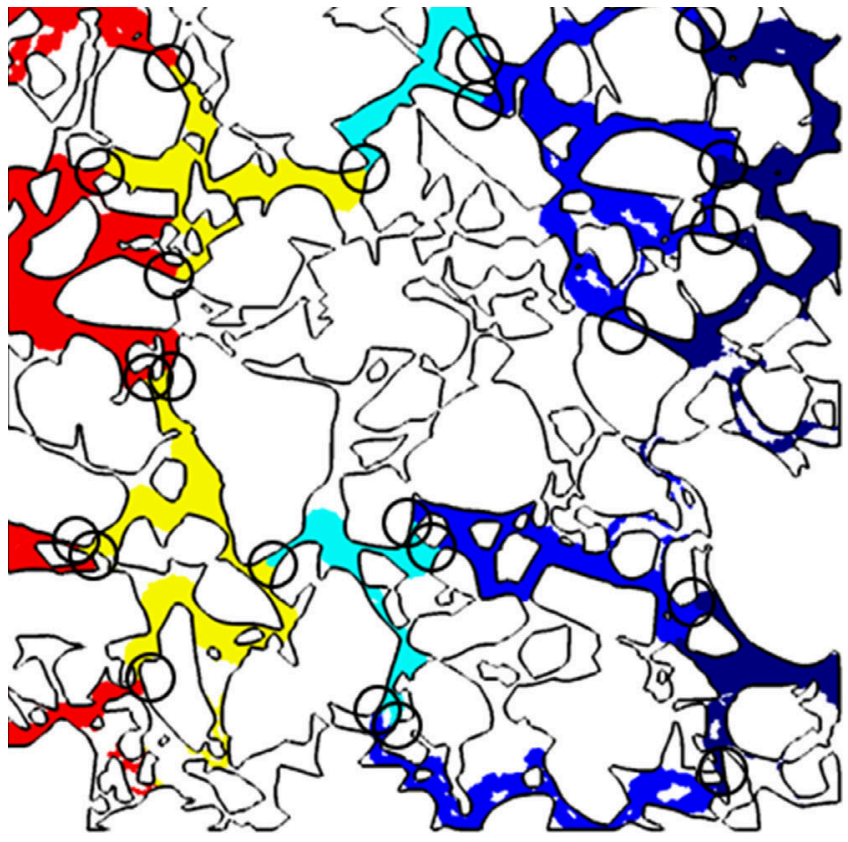

Fig. 9. Contour map showing 5 different levels of pressure within the sand pack for a pressure difference of $4000 \mathrm{~Pa}$ applied between the ends of the medium. Circles are used to highlight the pore constrictions in which most pressure loss is produced. Dark blue represents 0 - $460 \mathrm{~Pa}$, light blue corresponds to $460 \mathrm{~Pa}-1480$ $\mathrm{Pa}$, greenish blue covers $1480 \mathrm{~Pa}-2500 \mathrm{~Pa}$, yellow corresponds to the range 2500 $\mathrm{Pa}-3520 \mathrm{~Pa}$ and red colour represents $3520 \mathrm{~Pa}-4000 \mathrm{~Pa}$. White zones represent stagnant pores and solid grains. (For interpretation of the references to color in this figure legend, the reader is referred to the web version of this article.)

by $\mathrm{T}$, a value of $27 \mu \mathrm{m}$ is obtained, which coincides with the average pore size provided by YSM in this medium. This result must be further assessed, for different porous media and boundary conditions, before drawing definite conclusions. Nevertheless, the analysis method presented here is expected to be a valuable tool for future steps in that direction.

Previous results were published in which YSM was used to characterize the PSD cores of highly permeable sandstones and synthetic filters (Rodríguez de Castro et al., 2014, 2016, 2018). Despite xanthan gum polymer solutions being appropriate to investigate such microporous materials, a fundamental task for future experimental studies consists in selecting a suitable injection fluid to experimentally characterize low-permeability media and nanoporous materials without significant alteration in the morphology of the porous medium (due to particle or polymer retention). The technical specifications of the standard experimental setup used in YSM laboratory tests can also be consulted in the preceding works, e.g., the experimental uncertainties and the working ranges of the equipment.

\section{Conclusions and prospects}

Three techniques to extract PSDs from a digital image of a porous medium have been presented in the current work, including the numerical versions of Mercury Injection Porosimetry (MIP) and Yield Stress fluid injection Method (YSM). Pursuing the major objective of characterising the relevant pore dimensions controlling the relationship between injection flow rate and pressure gradient, the efficacy of the two porosimetry methods has been assessed through comparison to the equivalent pore networks models of three 2D porous media of increasing geometrical and morphological complexity. Good agreement was observed between the results obtained with the different approaches. In this regard, MIP and YSM provide pore dimensions that are closer to the throat sizes than to the body sizes, as expected given the principle of the measurements.

YSM has been shown, not only to be an effective experimental alternative to toxic MIP, but also a novel benchmark to analyse the images provided by micro-tomography. Furthermore, the PSDs obtained by YSM can be reliably used to predict the dependence of flow rate on pressure gradient by using the available analytical formulas for the flow of both Newtonian and non-Newtonian fluids through rectangular channels of infinite width. Also, numerical simulations have been confirmed as a powerful tool to investigate the mechanisms affecting micro-scale flow of yield stress fluids in 2D porous media and, in particular, to illustrate and validate the physical principle of YSM.

Despite being based on a bundle-of-capillaries model, YSM provides representative PSDs for $2 \mathrm{D}$ porous media with realistic topologies and morphologies. Therefore, considerable progress has been made towards proving the applicability of this technique to characterize porous media used in diverse industrial applications. These conclusions must now be extended to the case of 3D porous media, which will demand considerable computational power. In this regard, a possible alternative to direct simulation consists in calculating the pressure and velocity maps from the extracted pore network. This will imply accurate modelling of the shear viscosity of the fluid through the throats of such network.

\section{Declaration of Competing Interest}

This research did not receive any specific grant from funding agencies in the public, commercial, or not-for-profit sectors.

\section{References}

Abou Najm, M.R., Atallah, N.M., 2016. Non-Newtonian fluids in action: revisiting hydraulic conductivity and pore size distribution of porous media. Vadose Zone J. $15,1539-1663$.

Agnaou, M. Lasseux, D. Ahmadi, A., 2016. From steady to unsteady laminar flow in model porous structures: an investigation of the first Hopf bifurcation. Comput. Fluids 136, 67-82

Agnaou, M., Lasseux, D., Ahmadi, A., 2017. Origin of the inertial deviation from Darcy's law: an investigation from a microscopic flow analysis on two-dimensional model structures. Phys. Rev. E 96, 043105.

Alfi, M., Barrufet, M., Killough, J., 2019. Effect of pore sizes on composition distribution and enhance recovery from liquid shale - Molecular sieving in low permeability reservoirs. Fuel 235, 1555-1564.

Ambari, A., Benhamou, M., Roux, S., Guyon, E., 1990. Distribution des tailles des pores d'un milieu poreux déterminée par l'écoulement d'un fluide à seuil. Comptes rendus de l'Académie des Sci. 311 (II), 1291-1295.

Amirtharaj, E.S., Ioannidis, M.A., Parker, B., Tsakiroglou, C.D., 2011. Statistical synthesis of imaging and porosimetry data for the charasterization of microstructure and transport properties of sandstones. Transp. Porous Media 86, 135154.

Arns, C.H., 2004. A comparison of pore size distributions derived by NMR and X-ray-CT techniques. Phys. A 339, 159-165

Atallah, N.M., Abou Najm, M.R., 2019. Characterization of synthetic porous media using non-Newtonian fluids: experimental device. Eur. J. Soil Sci. 70, 257-267.

Balhoff, M., Sanchez-Rivera, D., Kwok, A., Mehmani, Y., Prodanovic, M., 2012. Numerical algorithms for network modeling of yield stress and other non-Newtonian fluids in porous media. Transp. Porous Media 93, 363-379.

Bao, K., Lavrov, A., Nilsen, H.M., 2017. Numerical modeling of non-Newtonian fluid flow in fractures and porous media. Comput. Geosci. 21, 1313-1324.

Bultreys, T., De Boever, W., Cnudde, V., 2016. Imaging and image-based fluid transport modeling at the pore scale in geological materials: a practical introduction to the current state-of-the-art. Earth Sci. Rev. 155, 93-128.

Burlion, N., Bernard, D., Chen, D., 2006. X-ray microtomography, application to microstructure analysis of a cementitious material during leaching process. Cem. Concr. Res. 36, 346-357.

Caulkin, R., Islam, M.S., Jia, X., Fairweather, M., 2014. Studies for the development of a virtual permeameter. Comput. Chem. Eng. 68, 190-202.

Chauveteau, G., Nabzar, L., El Attar, L., Jacquin, C., 1996. Pore structure and hydrodynamics in sandstones. In: Proceedings of the SCA Conference Paper Number p. 9607.

Chen, G., Lu, S., Zhang, J., Pervukhina, M., Liu, K., Wang, M., Han, T., Tian, S., Li, J., Zhang, Y., Ch, Xu., 2018. A method for determining oil-bearing pore size distribution in shales: a case study from the Damintun Sag, China. J. Petrol. Sci. Eng. 166, 673-678. 
Chevalier, T., Talon, L., 2015. Generalization of Darcy's law for Bingham fluids in porous media: from flow-field statistics to the flow-rate regimes. Phys. Rev. E 91, 023011.

Cieszko, M., Kempinski, M., Czerwinski, T., 2019. Limit models of pore space structure of porous materials for determination of limit pore size distributions based on mercury intrusion data. Transp. Porous Media 127, 433-458.

Coates, G.R., Xiao, L., Prammer, M.G., 1999. NMR Logging. Principles and Applications. Halliburton Energy Services, Houston.

Ferrás, L.L., Nóbrega, J.M., Pinho, F.T., 2012. Analytical solutions for Newtonian and inelastic non-Newtonian flows with wall slip. J. Non Newton. Fluid Mech. 175-176, 76-88.

Giesche, H., 2006. Mercury porosimetry: a general (practical) overview. Part. Part Syst. Charact. 23, 1-11.

Good, R.J., Mikhail, R.S., 1981. The contact angle in mercury intrusion porosimetry. Powder Technol. 19, 53-62.

Gostick, J., Aghighi, M., Hinebaugh, J., Tranter, T., Hoeh, M.A., Day, H., Spellacy, B., Sharqawy, M.H., Bazylak, A., Burns, A., Lehnert, W., 2016. OpenPNM: a pore network modeling package. Comput. Sci. Eng. 18, 60-74.

Gostick, J.T., 2017. Versatile and efficient pore network extraction method using marker-based watershed segmentation. Phys. Rev. E 96, 023307.

Gostick, J.T., Khan, Z.A., Tranter, T.G., Kok, M.D., Agnaou, M., Sadeghi, M., Jervis, R., 2019. PoreSpy: a python toolkit for quantitative analysis of porous media images. J. Open Source Softw. 4, 1296.

Guan, K.M., Nazarova, M., Guo, B., Tchelepi, H., Kovscek, A.R., Creux, P., 2019. Effects of image resolution on sandstone porosity and permeability as obtained from X-Ray microscopy. Transp. Porous Media 127, 233-245.

Herschel, W.H., Bulkley, R., 1926. Konsistenzmessungen von Gummi-Benzollösungen. Kolloid-Zeitschrift 39, 291-300.

Houston, A.N., Otten, W., Falconer, R., Monga, O., Baveye, P.C., Hapca, S.M., 2017. Quantification of the pore size distribution of soils: assessment of existing software using tomographic and synthetic 3D images. Geoderma 299, 73-82.

Hunt, A.G., 2001. Applications of percolation theory to porous media with distributed local conductances. Adv. Water Resour. 24, 279-307.

Hunt, A.G., Gee, G.W., 2002. Applications of critical path analysis to fractal porous media: comparison with examples from the HANford site. Adv. Water Resour. 25, 129-146.

Hyväluoma, J., Raiskinmäki, P., Jäsberg, A., Koponen, A., Kataja, M., Timonen, J., 2004. Evaluation of a lattice-Boltzmann method for mercury intrusion porosimetry simulations. Future Gener. Comput. Syst. 20, 1003-1011.

Khan, Z.A., Tranter, T., Agnaou, M., Elkamel, A., Gostick, J., 2019. Dual network extraction algorithm to investigate multiple transport processes in porous materials: image-based modeling of pore and grain scale processes. Comput. Chem. Eng. 123, 64-77.

León, Y., León, C.A., 1998. New perspectives in mercury porosimetry. Adv. Colloid Interface Sci. 76-77, 341-372.

Li, X., Kang, Y., Haghighi, M., 2018. Investigation of pore size distributions of coals with different structures by nuclear magnetic resonance (NMR) and mercury intrusion porosimetry (MIP). Measurements 116, 122-128.

Lindquist, W.B., Venkatarangan, A., 2000. Pore and throat size distributions measured from synchrotron X-ray tomographic images of Fontainebleau sandstones. J. Geophys. Res. Solid Earth 105 (B9), 21509-21527.

Malvault, G., Ahmadi, A., Omari, A, 2017. Numerical simulation of yield stress fluid flow in capillary bundles: influence of the form and the axial variation in the cross section. Transp. Porous Media 120, 255-270.

Malvault, G., 2013. Détermination expérimentale de la distribution de taille de pores d'un milieu poreux par l'injection d'un fluide à seuil ou par analyse fréquentielle, PhD thesis, Arts et Métiers ParisTech.

Minagawa, H., Nishikawa, Y., Ikeda, I., Miyazaki, K., Takahara, N., Sakamoto, Y., Komai, T., Narita, H., 2008. Characterization of sand sediment by pore size distribution and permeability using proton nuclear magnetic resonance measurement. J. Geophys. Res. 113, B07210.

Molnar, I., 2016. Uniform quartz - Silver nanoparticle injection experiment. Digital Rocks Portal. [Online]. Available: http://www.digitalrocksportal.org . [Assessed: 5-Feb-2019].

Muller, A.C.A., Scrivener, K.L., 2017. A reassessment of mercury intrusion porosimetry by comparison with ${ }^{1} \mathrm{H}$ NMR relaxometry. Cem. Concr. Res. 100, 350-360.

Oukhlef, A., 2011. Détermination de la distribution de tailles de pores d'un milieu poreux (PhD thesis). Arts et Métiers ParisTech.

Oukhlef, A., Champmartin, S., Ambari, A., 2014. Yield stress fluids method to determine the pore size distribution of a porous medium. J. Non Newton. Fluid Mech. 204, 87-93.

Ouyang, L., Hill, A.D., Zhu, D., 2013. Theoretical and numerical simulation of Herschel-Bulkley fluid flow in propped fractures. Soc. Petrol. Eng. doi:10.2118/ 163841-MS.

Papanastasiou, T.C., 1987. Flow of materials with yield. J. Rheol. 31, 385-404.

Paul, S., Roy, S., Ghosh, P., Faghihi Zarandi, M.A., Cender, T., Pillai, K.M., 2019. A novel method for permeability estimation from micro-tomographic images. Transp. Porous Media 127, 127-171.

Payatakes, A.C., Dias, M.M., 1984. Immiscible microdisplacement and ganglion dynamics in porous media. Rev. Chem. Eng. 2, 85-174.

Prashant, Derksen J.J., 2011. Direct simulations of spherical particle motion in Bingham liquids. Comput. Chem. Eng. 35, 1200-1214.
Rodríguez de Castro, A., Ahmadi-Sénichault, A., Omari, A., 2018. Using xanthan gum solutions to characterize porous media with the yield stress fluid porosimetry method: robustness of the method and effects of polymer concentration. Transp. Porous Media 122, 357-374.

Rodríguez de Castro, A., Omari, A., Ahmadi-Sénichault, A., Bruneau, D., 2014. Toward a new method of porosimetry: principles and experiments. Transp. Porous Media 101, 349-364.

Rodríguez de Castro, A., Omari, A., Ahmadi-Sénichault, A., Savin, S., Madariaga, L.-F., 2016. Characterizing porous media with the yield stress fluids porosimetry method. Transp. Porous Media 114, 213-233.

Roels, S., Elsen, J., Carmeliet, J., Hens, H., 2001. Characterisation of pore structure by combining mercury porosimetry and micrography. Mater. Struct./Matériaux et Constr. 34, 76-82.

Rouquerol, J., Baron, G., Denoyel, R., Giesche, H., Groen, J., Klobes, P., Levitz, P. Neimark, A.V., Rigby, S., Skudas, R., Sing, K., Thommes, M., Unger, K., 2012. Liquid intrusion and alternative methods for the characterization of macroporous materials (IUPAC Technical Report). Pure Appl. Chem. 84, 107-136.

Salejova, G., Grof, Z., Solcova, O., Schneider, P., Kosek, J., 2011. Strategy for predicting effective transport properties of complex porous structures. Comput. Chem. Eng. $35,200-211$.

Saramito, P., Wachs, A., 2017. Progress in numerical simulation of yield stress fluid flows. Rheol. Acta 56, 211-230.

Saxena, N., Hows, A., Hofmann, R., Alpak, F.O., Freeman, J., Hunter, S., Appel, M., 2018. Imaging and computational considerations for image computed permeability: operating envelope of digital rock physics. Adv. Water Resour. 116, 127-144.

Schenk, O., Gärtner, K., 2004. Solving unsymmetric sparse systems of linear equations with PARDISO. Future Gener. Comput. Syst. 20, 475-487.

Shah, C.B., Yortsos, Y.C., 1995. Aspects of flow of power-law fluids in porous media. AlChE J. 41, 1099-1112.

Sheppard, A., Prodanovic, M., 2015. Network generation comparison forum. Digit Rocks Portal. [Online]. Available: http://www.digitalrocksportal.org . [Assessed: 5-Feb-2019].

Sheppard, A.P., Sok, R.M., Averdunk, H., 2004. Techniques for image enhancement and segmentation of tomographic images of porous materials. Phys. A Stat Mech. Appl. 339, 145-151.

Skelland, A.H.P., 1967. Non-Newtonian Flow and Heat Transfer. Wiley, New York.

Suresh Kumar, P., Korving, L., Keesman, K.J., van Loosdrecht, M.C.M., Witkamp, G.-J., 2019. Effect of pore size distribution and particle size of porous metal oxides on phosphate adsorption capacity and kinetics. Chem. Eng. J. 358, 160-169.

Tosco, T., Marchisio, D.L., Lince, F., Sethi, R., 2013. Extension of the Darcy-Forchheimer law for shear-thinning fluids and validation via pore-scale flow simulations. Transp. Porous Media 96, 1-20.

Tsakiroglou, C.D., 2002a. Determination of the transport properties of single fractures with the aid of critical path analysis. Ind. Eng. Chem. Res. 41, 3462-3472.

Tsakiroglou, C.D., 2002b. A methodology for the derivation of non-Darcian models for the flow of generalized Newtonian fluids in porous media. J. Non Newton. Fluid Mech. 105, 79-110.

Tsakiroglou, C.D., 2004. Correlation of the two-phase flow coefficients of porous media with the rheology of shear-thinning fluids. J. Non Newton. Fluid Mech. 117, $1-23$.

Tsakiroglou, C.D., Payatakes, A.C., 2000. Characterization of the pore structure of reservoir rocks with the aid of serial sectioning analysis, mercury porosimetry and network simulation. Adv. Water Resour. 23, 773-789.

Tsakiroglou, C.D., Ioannidis, M.A., 2008. Dual-porosity modelling of the pore structure and transport properties of a contaminated soil. Eur. J. Soil Sci. 59, 744-761.

Tsakiroglou, C.D., Ioannidis, M.A., Amirtharaj, E., Vizika, O., 2009. A new approach for the characterization of the pore structure of dual porosity rocks. Chem. Eng. Sci. 64, 847-859.

United Nations, 2013. United Nations Environment Programme. Text of the Minamata Convention on Mercury for adoption by the Conference of Plenipotentiaries. The Conference of Plenipotentiaries on the "Minamata Convention on Mercury". http://www.mercuryconvention.org/ (Accessed 05.03.2019).

Van Brakel, J. (Ed.), 1981. A special issue devoted to mercury porosimetry. Powder Technol. 29.

Wang, X., Hou, J., Song, S., Wang, D., Gong, L., Ma, K., Liu, Y., Liu, Y., Li, Y., Yan, L. 2018. Combining pressure-controlled porosimetry and rate-controlled porosimetry to investigate the fractal characteristics of full-range pores in tight oil reservoirs. J. Petrol. Sci. Eng. 171, 353-361.

Washburn, E.W., 1921. The dynamics of capillary flow. Phys. Rev, 17, 273-283.

Wu, J., Feng, M., Mao, X., Xu, J., Zhang, W., Ni, X., Han, G., 2018. Particle size distribution of aggregate effects on mechanical and structural properties of cemented rockfill: Experiments and modelling. Constr. Build. Mater. 193, 295-311.

Xiong, Q., Baychev, T.G., Jivkov, A.P., 2016. Review of pore network modelling of porous media: Experimental characterisations, network constructions and applications to reactive transport. Journal of Contaminant Hydrology 192, 101-117.

Zamani, N., Bondino, I., Kaufmann, R., Skauge, A., 2017. Computation of polymer in-situ rheology using direct numerical simulation. J. Petrol. Sci. Eng. 159, 92-102.

Zhang, L., Sun, H., Han, B., Peng, L., Ning, F., Jiang, G., Chehotkin, V.F., 2016. Effect of shearing actions on the rheological properties and mesostructures of CMC. PVP and $\mathrm{CMC}+\mathrm{PVP}$ aqueous solutions as simple water-based drilling fluids for gas hydrate drilling. J. Unconv. Oil Gas Resour. 14, 86-98. 